\title{
Copper Indium Disulfide Quantum Dot Light Emitting Diodes for Display and Lighting Technologies
}

\author{
Stefano Barba \\ Herbert Wertheim College of Engineering, University of Florida
}

Faculty mentor: Jiangeng Xue, Department of Materials Science and Engineering

\begin{abstract}
While significant advances in the development of quantum dot light emitting diodes (QLEDs) have been reported, these devices are primarily based on cadmium chalcogenide quantum dot (QD) materials. Both environmental and health concerns arise due to the toxicity of cadmium and consequently, alternative less toxic QDs must be developed for large scale QLED applications such as display and solid state lighting technologies. In this work, copper indium disulfide (CIS) was investigated as an alternative QD material for QLED applications. Through experimentation with material synthesis and device fabrication, this project aimed to develop high performing CIS QLEDs. Several synthetic approaches were experimented with and it was determined that the injection of shorter chain 1-octanethiol as the sulfur precursor with extensive shell reaction time resulted in highly luminescent QDs. Single color QLEDs were fabricated based on typical device structure, using highly luminescent synthesized CIS QDs as the emissive layer in multilayer devices. Varying the shell reaction time of QDs in order to vary shell thickness resulted in significant differences in device performance. Using thicker shell QDs, high performing devices were obtained with the best performing QLEDs displaying a high peak current efficiency of $14.7 \mathrm{~cd} / \mathrm{A}$ and high external quantum efficiency of $5.2 \%$.
\end{abstract}

Keywords: quantum dots, diodes, copper indium disulfide

\section{Introduction}

Semiconductor quantum dots (QDs) are among the most researched materials due to the wide variety of potential applications for these materials which include bio-imaging [1], sensors [2], lasers [3], and light emitting diodes (LEDs) [4] . Consisting of semiconductor nanocrystals that contain only a few atoms and range from 2 to $15 \mathrm{~nm}$ in size [5], these materials have properties that differ significantly from their bulk counterpart. Unique optical properties such as size controlled tunable emission wavelengths, narrow emission spectrums and high luminescent efficiency [6] all contribute to the versatility and potential applications of these materials.

Since their discovery and subsequent development, one of the main focuses for QDs has been their application as emitting materials in light emitting diodes (LEDs). QDs are favorable candidates for next generation display and solid-state lighting technologies since they are able 
emit bright, pure, and size tunable light in visible spectrum with the added advantage of solution processability [7]. In fact, quantum dot-based displays, which feature QDs as color converters for LCD backlights are already on the market and a main goal of the industry is to achieve high performing true quantum dot light emitting diode (QLED) displays. It was forecast that the overall market for QLEDs in displays and lighting will be able to reach \$10.8 billion by 2026 [8] and thus developments in this technology have a clear economic incentive.

QLED technology has undergone significant advances since its earliest generation which consisted of bilayers or blends of QDs and polymers [9]. These QLEDs were limited in performance because QDs performed both light emission and charge conduction. Later QLEDs fabricated by layering emissive layers (EMLs) of QDs in between two organic charge transport layers showed significant performance improvement due to the more efficient injection of charge into the QDs. Current QLED technology consist of multilayered devices where a combination of inorganic electron transport layers (ETLs), organic hole injection layers (HILs), and organic hole transport layers (HTLs) are used in order to obtain highly efficient devices. These layers significantly improve device performance by optimizing charge movements so that recombination can occur at the QD layer [10].

Currently, the best reported QLEDs are primarily fabricated with cadmium-based quantum dots. Cadmium QDs provide significant advantages due to their high photoluminescent efficiencies and narrow emissions. Using CdZnSeS QDs and optimized tandem structures, Chen's group was able to fabricate green QLEDs exhibiting record high current efficiencies $\left(\eta_{A}\right)$ over $100 \mathrm{~cd} / \mathrm{A}$ and an external quantum efficiencies $\left(\eta_{\mathrm{EQE}}\right)$ over 23\% [11]. While these devices have reached impressive performance levels, they are limited by the health and environmental concerns raised by the use of toxic cadmium. The European Union has already set limits on cadmium concentrations in electronics through the RoHS directive [12] and further policy setting organizations are expected to further limit cadmium use in the future. Thus, large-scale applications of QLEDs must be developed to be cadmium free.

Copper indium disulfide (CIS) is semiconductor material with certain optical properties that make it a promising alternative to cadmium-based materials for QDs. CIS has a direct band gap with a bulk value of $1.45 \mathrm{eV}$ and high photostability. CIS QDs have been reported to be have tunable emissions from the near infrared to visible range, high quantum yields, large Stokes shift, and large luminescence decay time [13]. Thus, this material system has created significant 
interest due to the possible applications and reduced toxicity. CIS has high defect tolerance and frequently varies from exact 1:1:2 ratios. Because of this, radiative and non-radiative charge combinations are attributed to defects within CIS rather than direct recombination paths. These indirect recombination routes lead to a broad emission spectrum compared to cadmium based QDs. Moreover, the defect related emission is also attributed to lower photoluminescent efficiencies compared to other QDs. Combined with the difficult nature of controlling precursor reactivity in ternary compounds, synthetic methods for CIS QDs continue to be explored in order to optimize CIS QDs and bypass some of their intrinsic limitations.

Fabrication of functioning CIS QLEDs have been reported, but the performance of these devices still lag significantly behind other QLED technology with all reported $\eta_{\mathrm{EQE}}$ values for CIS QLEDs being below 10\%. CIS QLED technology must continue to be developed and optimized before it can be viable for any large-scale application. This project was motivated by these challenges and sought to contribute to the development of CIS QLEDs. The main goals of this project were to: (1) investigate and experiment with different synthetic methods for CIS QDs to optimize the photoluminescent properties of synthesized QDs, (2) fabricate high performing CIS QLEDs, and (3) investigate different ways to optimize device performance. Through modulation of these parameters, it was believed that devices could be fabricated to obtain $\eta_{\mathrm{EQE}}$ values above $10 \%$.

\section{Methods}

\section{Synthesis of CuInS2 Quantum Dots}

Heating up and hot injection synthesis methods were explored in order to synthesize CIS QDs. Various synthetic parameters and methods for making CIS QDs were used in order to optimize luminescent behavior of made QDs. In all these methods, QDs were solution processed using a three-neck flask, stir bar, condenser, temperature sensor, and heating mantel. A Schleck line was used in order to degas the solution and provide continuous nitrogen gas flow into the reaction. Heating-up synthesis required placing all molecular precursors and solvents into the reaction vessel and then heating the solution up to promote nucleation of QDs. The hot injection method consisted of mixing certain precursors and solvents into the reaction vessel, heating that solution, and then injecting another precursor into the heated solution. QDs were then purified three times by their precipitation in excess of acetone using centrifugation (6000 RPM, 5 minutes) and redispersion in chloroform. For the synthesis of CIS/ZnS QDs, ZnS precursors 
were dissolved in organic solvents and then injected into the QD solution once they had reacted for the desired time. For the ligand exchange process of QDs, a $5 \mathrm{ml}$ solution of obtained QDs was purified once and then redispered in chloroform. $3 \mathrm{ml}$ of 1-octanethiol (OTT) were added to the solution and the subsequent solution was stirred vigorously for 5 hours.

\section{Device Fabrication}

Devices were fabricated using typical device structures for QLEDs shown in figure 1. Patterned Indium Tin Oxide (ITO) coated glass substrates with a sheet resistance of around 20 $\Omega$-sq ${ }^{-1}$ were used as the base of the device. Substrates were cleaned through sonication using a solution of soap and water followed by deionized water, acetone, and isopropanol all consecutively for 15 minutes each. Substrates where then furthered cleaned using ozone generated by ultraviolet light in air. Poly(3,4-ethylenedioxythiophene) polystyrene sulfonate (PEDOT:PSS) (AI 4083) was spin coated onto the substrate at 3000 RPM for 30 seconds and then baked in air at $150^{\circ} \mathrm{C}$ for 15 minutes. Substrates where then moved to a nitrogen gas filled glove box in order to spin coat the sequential device layers. Poly [9,9-dioctylfluorene-co-N-[4(3-methylpropyl)]-diphenylamine] (TFB) in chlorobenzene $(30 \mathrm{mg} / \mathrm{ml})$ was spin coated onto the substrates at 3000 RPM for 30 seconds and then baked at $150^{\circ} \mathrm{C}$ for 15 minutes. This was followed by spin coating of the QD solution in which concentration and solvent were varied for experimental purposes. The QD layers were spin coated at 2000 RPM for 30 seconds and baked at $60^{\circ} \mathrm{C}$ for 15 minutes.

Zinc Oxide $(\mathrm{ZnO})$ nanoparticles were synthesized earlier by the dropwise addition of a stoichiometric amount of tetramethylammonium hydroxide (TMAH) dissolved in ethanol (0.55 $\mathrm{M}$ ) to $30 \mathrm{ml}$ of $0.1 \mathrm{M}$ zinc acetate dihydrate dissolved in dimethyl sulfoxide (DMSO) followed by stirring for an hour at room temperature. $\mathrm{ZnO}$ nanoparticles were then purified and dispersed in ethanol with a concentration of $\sim 30 \mathrm{mg} / \mathrm{ml}$. The $\mathrm{ZnO}$ nanoparticles in ethanol were spin coated at 2000 RPM for 30 seconds and then baked at $60^{\circ} \mathrm{C}$ for 15 minutes. The samples were then loaded into a high-vacuum thermal deposition chamber in order to deposit an aluminum cathode (100 nm thick) patterned by an in situ shadow mask to form an active device area of 4 $\mathrm{mm}^{2}$. 


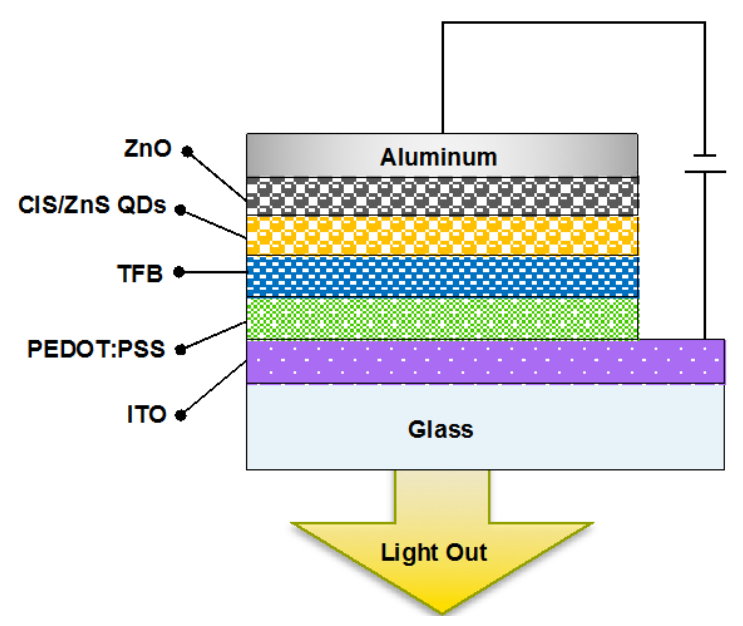

Figure 1. Device structure for fabricated QDs

\section{Characterization}

Absorption spectra were obtained using an Orial Apex monochromatic beam chopped at 380 Hz. A calibrated silicon detector (Newport 818 UV) was placed behind a sample holder in order to measure the transmittance of light through samples. Room temperature photoluminescent spectra were obtained using a Jasco spectrofluorometer (model FP-6500). Transmission electron microscopy (FEI TECNAI F20) was used to image QDs and determine their average diameter.

In order to measure the photoluminescent quantum yield ( $\left.\eta_{\mathrm{QY}}\right)$ of QDs, the ratio of photons emitted to photons absorbed, the comparative method developed by Williams et A1 [14] was used. Cresyl violet dissolved in methanol and fluorescein dissolved 0.1M sodium hydroxide were used as standard for dark red and yellow-orange emitting QDs, respectively. In this method several low concentration solutions are made by dilution and recording of absorbance values. Emission spectra are then measured and integrated to create a plot of integrated fluorescence intensity over absorbance. This can then be used in order determine the $\eta_{Q Y}$ of samples by using the following correlation:

$$
\eta_{x}=\eta_{S t}\left(\frac{m_{x}}{m_{S t}}\right)\left(\frac{N_{x}}{N_{S t}}\right)^{2}
$$

where $\eta, \mathrm{m}$, and $\mathrm{N}$ are the quantum yield of emissive material, gradient of the plot, and refractive index of the solvent respectively. The subscript 'St' denotes the standard and the subscript ' $\mathrm{X}$ ' denotes the sample. Radiant Emittance (R) - Luminance (L) - Current density (J) Voltage (V) characteristics and electroluminescent (EL) spectrums of QLEDs were measured 
using an Agilent 4155C semiconductor parameter analyzer and a calibrated silicon detector (Newport 818 UV).

\section{Results}

\section{Overview of synthetic methods used for core CIS QDs}

Initial attempts to synthesize CIS QDs focused on the heating-up. Different reported recipes were used and modified in order to create several batches of CIS QDs. Initial findings showed poor luminescent behavior with the core QDs obtaining $\eta_{\mathrm{QY}}$ below 1\%. These procedures consisted of different copper and indium precursors, with dodecanthiol (DDT) acting as the sole source of sulfur and capping ligands in the reaction. Varying parameters such as reaction time, reaction temperature and precursor concentration had little effect on the $\eta_{\mathrm{QY}}$ of the QDs.

From this data, it was hypothesized that DDT as the sole source of sulfur and ligands in the reaction was ineffective. It was also hypothesized that using a non-injection method could also be limiting the performance of the QDs. The next synthetic method attempted used sulfur powder dissolved in trioctylphosphine oxide (TOPO) in order to provide a secondary sulfur source apart from DDT. The hot injection method of this solution showed significantly improved results from the previous synthesis method, but still lacked in overall performance. Varying synthesis parameters, the highest obtained $\eta_{\mathrm{QY}}$ in these core QDs was 4\%.

Based off a reported synthesis method by Kim et al [15], QDs were synthesized by replacing DDT with shorter chained OTT as the source of sulfur and capping ligands. QDs made using this procedure showed a significant increase in $\eta_{\mathrm{QY}}$ and thus this synthetic approach was used for further optimization of QDs. Table 1 below provides a summary of the different synthetic approaches.

Table 1. Summary of Core QD Synthesis

\begin{tabular}{lcc}
\hline QD Synthesis Method & Core QD $\eta_{\mathrm{QY}}(\%)$ & Modified method based on reference \\
\hline Heating-up method of CuAc, In(Ac)3, DDT, ODE & $<1$ & {$[16]$} \\
Heating-up method CuI, In(Ac)3, DDT, ODE & $<1$ & {$[17]$} \\
Hot Injection of TOPO-S into CuAc, In(Ac) 3 , DDT & 2 to 4 & {$[1]$} \\
Hot Injection of OTT into CuI, In(Ac) $)_{3}$, ODE & 14 & {$[15]$} \\
\hline Ac $=$ Acetate, $O D E=$ Octadecene, & &
\end{tabular}




\section{ZnS Shelled CIS QDs}

Following the reported synthesis route with the new high performing QDs, a shelling procedure was followed in order to create a $\mathrm{ZnS}$ shell around the core QDs. In order to vary shell thickness, the reaction times of the procedure were varied in order to create two types of QDs with shell reaction times of 3 hours and 11 hours, respectively. The overcoating of the QDs with $\mathrm{ZnS}$ resulted in a significant increase in $\eta_{\mathrm{QY}}$ reaching up to $75 \%$ for 11 hour reaction and $69 \%$ for the 3 hour reaction. Figure 2 shows the slight blue shift of the absorption and emission spectra for the longer reaction, indicating the band gap widening of the QDs as a result of the increase in shell thickness. As expected due to the nature of CIS, the emission spectrums of these QDs are wide with full width half maximum (FWHM) values of $100 \mathrm{~nm}$ for both shell reaction times. These large results for FWHM limit the color purity of the QDs needed for display technologies and thus are more useful for lighting applications in their current state. In the future, emissions must be narrowed down for effective display applications. The TEM images shown in figure 3 show average QD diameter size to be $4.38 \mathrm{~nm}$ and $5.31 \mathrm{~nm}$ for 3 hour and 11 hour shells respectively, confirming the increase in shell thickness size.

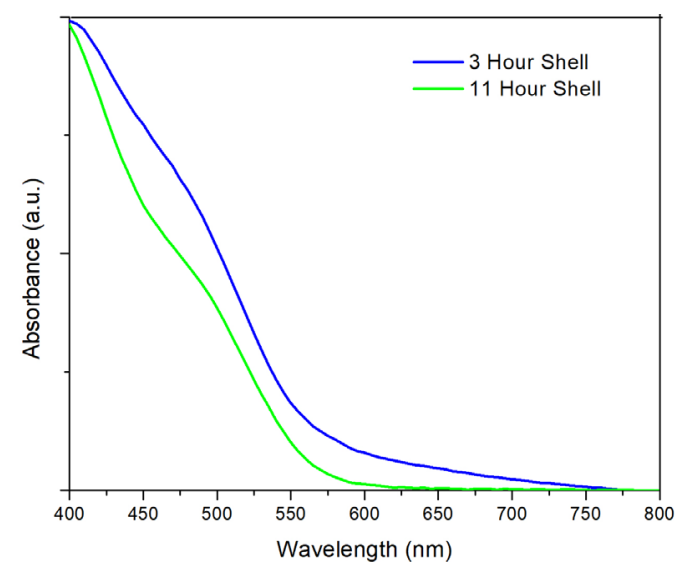

(a)

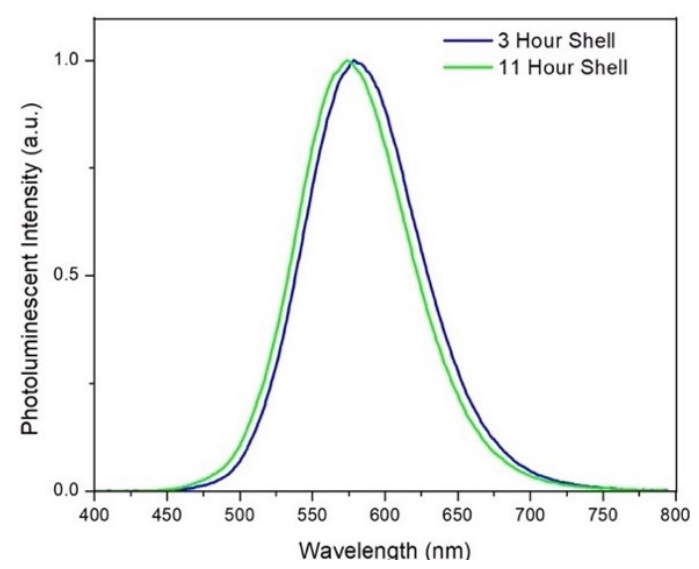

(b)

Figure 2. (a) Absorption and (b) PL spectra for the 3 hour and 11 hour shell reaction time QDs 


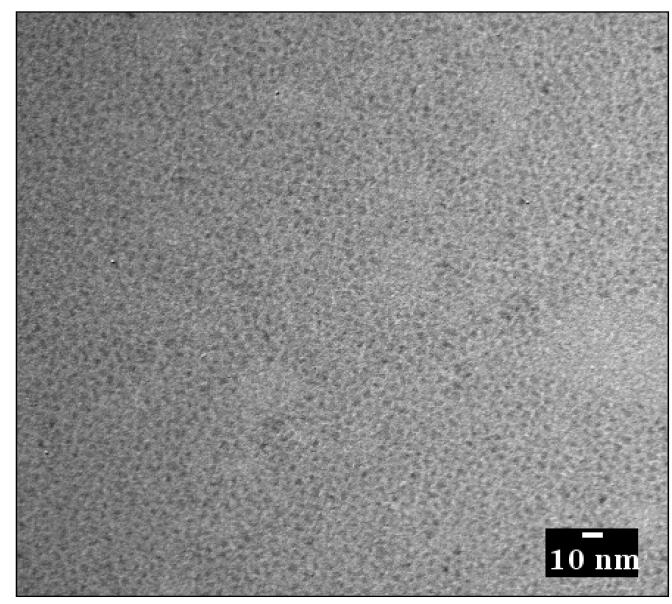

(a)

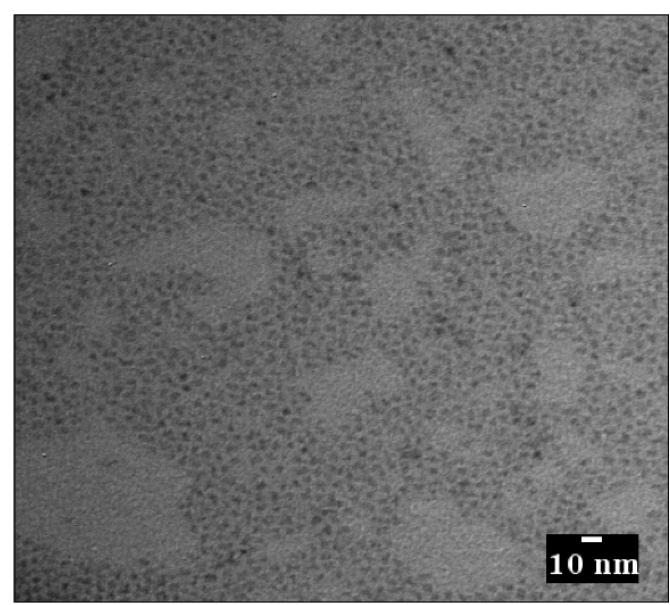

(b)

Figure 3. TEM images of CIS QDs for the (a) 3 hour shell and (b) 11 hour shell reaction time QDS

Devices were fabricated from these QDs due to the high $\eta_{\mathrm{QY}}$ obtained for both reactions. Figure 4 (a) shows how the longer shelled QDs display both higher luminance and higher current density levels than the shorter shell reactions. While it was expected that increasing shell thickness would create a larger barrier for current flow, the higher current densities indicate that this barrier is not significant at these shell thicknesses. It is assumed that the QDs with thicker shells have a more favorable core/shell interface that isolates the surface and prevents charge accumulation at the core layer. This in turn reduces non-radiative combination and results in improved properties.

Figure 4 (b) shows that the longer shell method was able to achieve both higher $\eta_{\mathrm{EQE}}$ and higher $\eta_{\mathrm{A}}$ values reaching peak values of $5.2 \%$ and $14.7 \mathrm{~cd} / \mathrm{A}$ which are significant values for CIS QLEDs close to highest reported values of 7\% and $18.2 \mathrm{~cd} / \mathrm{A}$ respectively [15] but still falling behind cadmium-based devices. The EL spectrum in figure 4(c) shows a slight red shift from the PL spectrum for both types of quantum dots. These devices emit yellow-orange light at a broad spectrum that slightly broadened from PL emission. Both devices had negative aging effects with reduction in $\eta_{\mathrm{EQE}}$ seen with each consecutive measurement day shown in figure 4(d). 


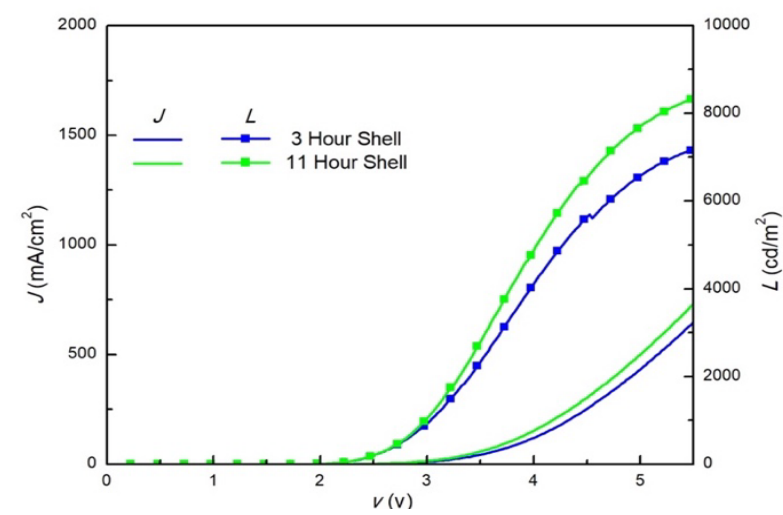

(a)

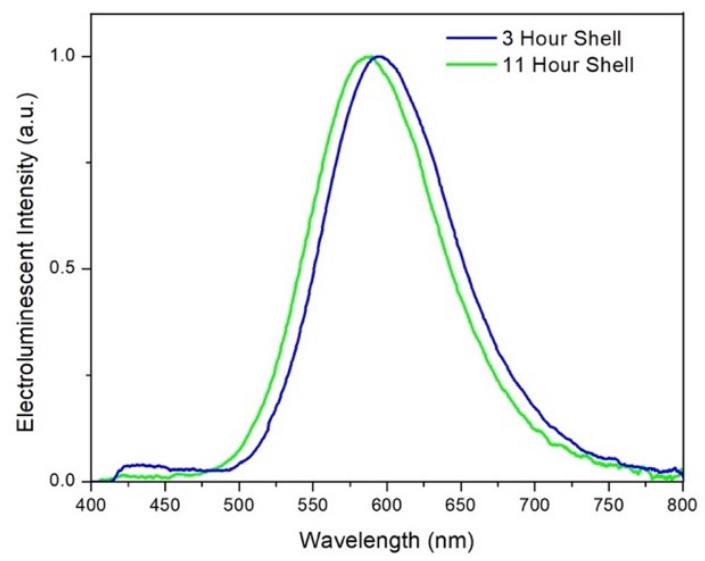

(c)

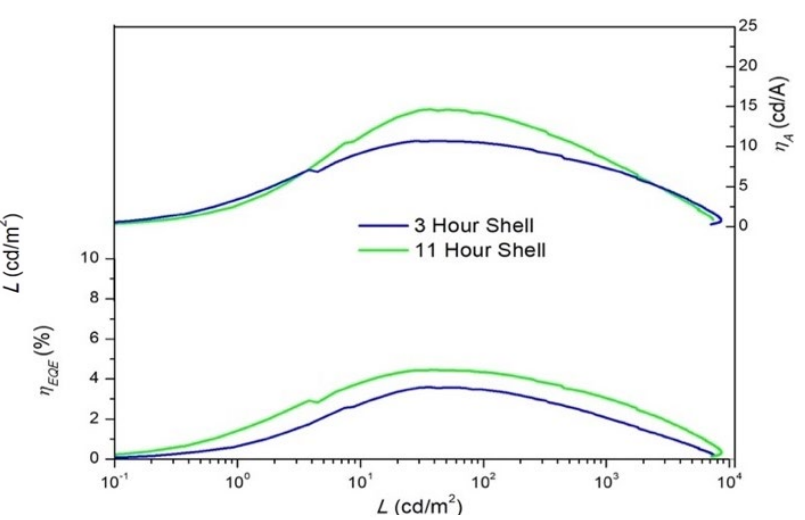

(b)

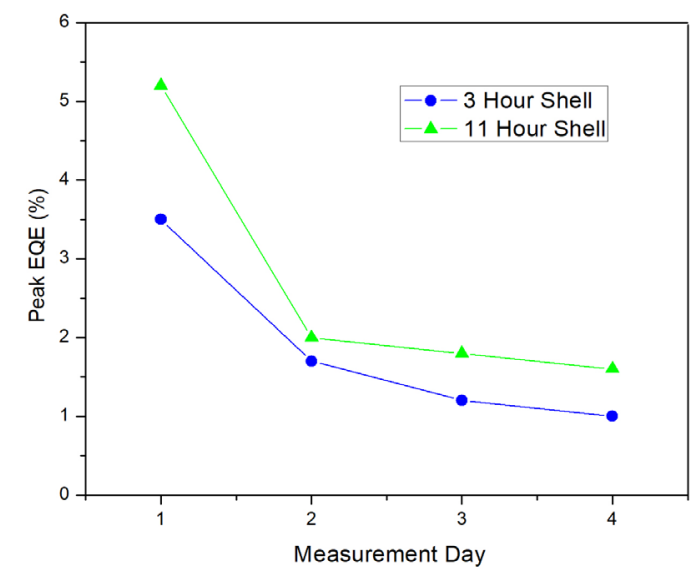

(d)

Figure 4. Device performance for the 3 hour and 11 hour shell reaction time QDs. (a) Current density and luminance as function of voltage, (b) current efficiency and external quantum efficiencies as a function of luminance and (c) the electroluminescent spectrum with the insert showing a device under applied voltage (d) Peak EQE changes over measurement time

Table 2. Comparison of emission peak wavelength $\lambda_{\max }, \mathrm{FWHM}$, turn-on voltage $\mathrm{V}_{\mathrm{T}}$, external quantum efficiency $\boldsymbol{\eta}_{\mathrm{EQE}}$, current efficiency $\boldsymbol{\eta}_{\mathrm{A}}$, and power efficiency $\boldsymbol{\eta}_{\mathrm{P}}$ of the two different shell reaction time QDs

\begin{tabular}{|c|c|c|c|c|c|c|c|c|c|}
\hline \multirow[t]{2}{*}{$\begin{array}{l}\text { Synthetic } \\
\text { method }\end{array}$} & \multirow[t]{2}{*}{$\lambda_{\max }(\mathrm{nm})$} & \multirow[t]{2}{*}{$\mathrm{FWHM}(\mathrm{nm})$} & \multirow[t]{2}{*}{$\mathrm{V}_{\mathrm{T}}(\mathrm{V})$} & \multicolumn{2}{|c|}{$\eta_{\mathrm{EQE}}(\%)$} & \multicolumn{2}{|c|}{$\begin{array}{l}\eta_{\mathrm{A}} \\
(\mathrm{cd} / \mathrm{A})\end{array}$} & \multicolumn{2}{|c|}{$\begin{array}{l}\eta_{\mathrm{P}} \\
(\mathrm{Lm} / \mathrm{W})\end{array}$} \\
\hline & & & & Peak & $\begin{array}{l}\text { at } 1000 \\
\mathrm{~cd} / \mathrm{m}^{2}\end{array}$ & Peak & $\begin{array}{l}\text { at } 1000 \\
\text { cd } / \mathrm{m}^{2}\end{array}$ & Peak & $\begin{array}{l}\text { at } 1000 \\
\mathrm{~cd} / \mathrm{m}^{2}\end{array}$ \\
\hline $\begin{array}{l}\text { Hour } \\
\text { Shell } \\
11 \text { Hour }\end{array}$ & 597 & 105 & 3.1 & 3.5 & 2.0 & 10.7 & 7.3 & 15.6 & 7.7 \\
\hline Shell & 590 & 102 & 3.6 & 5.2 & 3.0 & 14.7 & 8.4 & 20.9 & 8.6 \\
\hline
\end{tabular}

\section{Ligand Exchange}

In order to further optimize devices, a QD ligand exchange process for QDs was attempted in order replace the oleic acid ligands with shorter OTT ligands. Previous reports show that in CdS 
QDs, a ligand exchange caused a two-fold increase in electron mobility, which led to improved device performance [4]. The 11-hour shell method was used to synthesize new QDs from which two samples, a control solution and ligand exchange solution, were created. Only slight changes in QD properties were observed with the ligand exchange. Figure 5 (a) shows that with the ligand exchange came a slight red shift in the absorbance spectra and figure 5 (b) even smaller shift in the emission spectra.

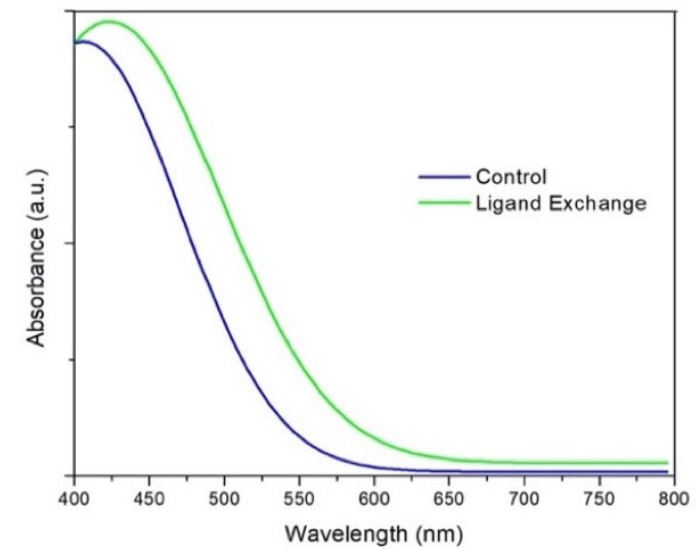

(a)

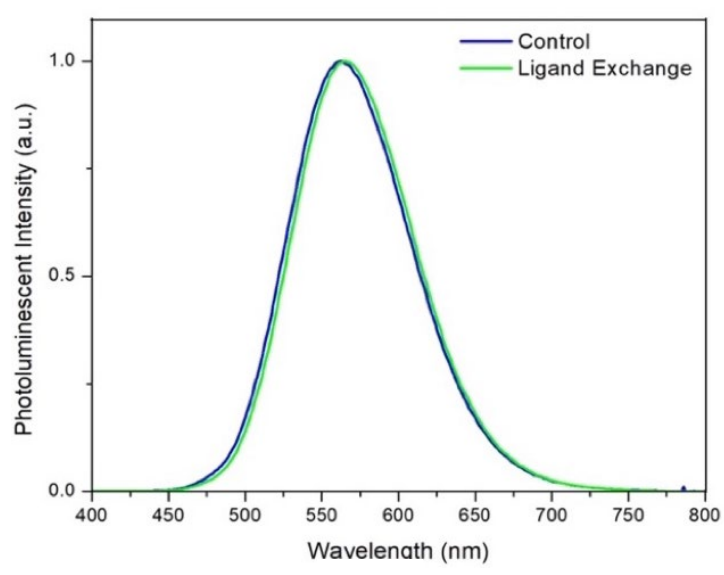

(b)

Figure 5. (a) Absorption and (b) PL spectra for the control and ligand exchange QDs

Devices made with these QDs also showed small differences in performance. Compared with earlier made devices, both samples performed significantly worse and that must be taken into account when discussing these results. One significant difference shown in figure 6(a) is the higher luminesce level obtained by the ligand exchange QDs. Apart from the luminance levels, performance levels for both devices only slightly deviated and both devices had nearly identical peak $\eta_{\mathrm{EQE}}$ and $\eta_{\mathrm{A}}$ values. The small differences in both QD and QLED indicate that there was ligand exchange to certain degree, however more analysis is needed in order to fully understand the effects of the ligand exchange in this material system. 


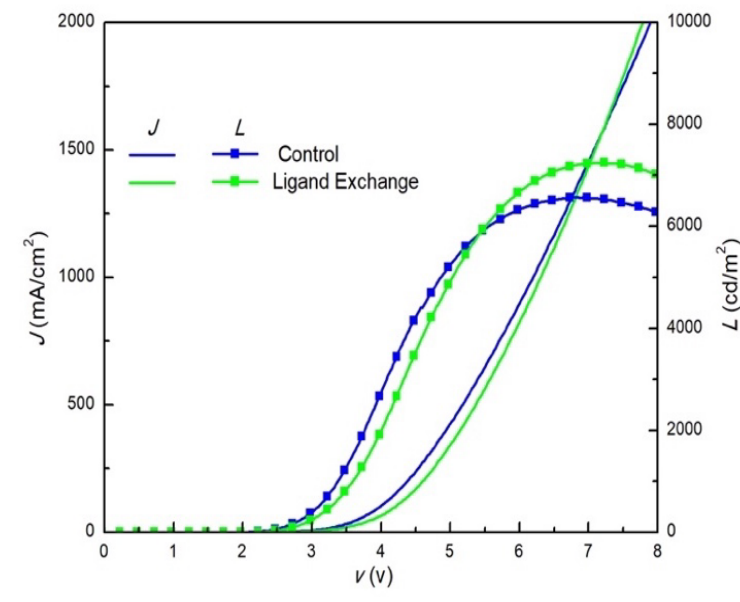

(a)

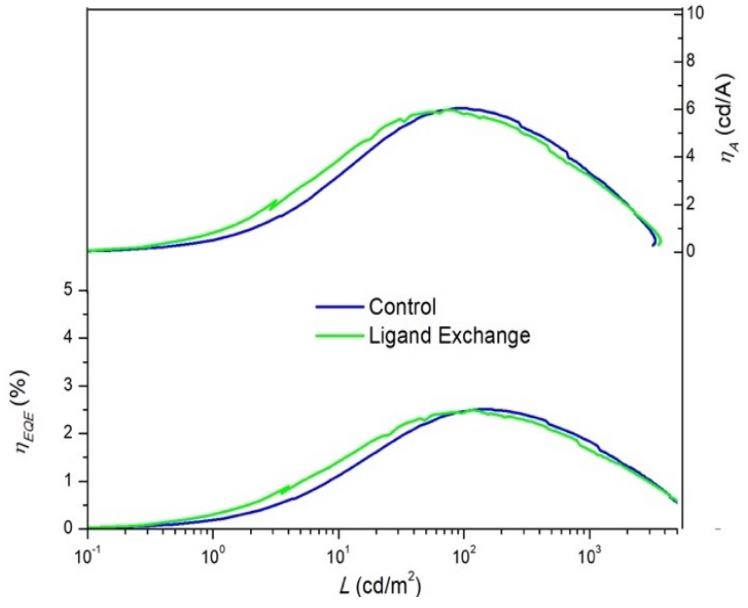

(b)

Figure 6. Device performance for the control and ligand exchange QDs. (a) Current density and luminance as function of voltage and (b) current efficiency and external quantum efficiencies as a function of luminance

Table 3. Comparison of turn-on voltage $\mathrm{V}_{\mathrm{T}}$, external quantum efficiency $\eta_{\mathrm{EQE}}$, current efficiency $\boldsymbol{\eta}_{\mathrm{A}}$, and power efficiency $\eta_{\mathrm{P}}$ of the CIS QLED control sample and with QD ligand exchange

\begin{tabular}{|c|c|c|c|c|c|c|c|}
\hline \multirow[t]{2}{*}{$\begin{array}{l}\text { Synthetic } \\
\text { method }\end{array}$} & \multirow[t]{2}{*}{$\mathrm{V}_{\mathrm{T}}(\mathrm{V})$} & \multicolumn{2}{|c|}{$\eta_{\mathrm{EQE}}(\%)$} & \multicolumn{2}{|l|}{$\begin{array}{l}\eta_{\mathrm{A}} \\
(\mathrm{cd} / \mathrm{A})\end{array}$} & \multicolumn{2}{|c|}{$\begin{array}{l}\eta_{\mathrm{P}} \\
(\mathrm{Lm} / \mathrm{W})\end{array}$} \\
\hline & & Peak & at $1000 \mathrm{~cd} / \mathrm{m}^{2}$ & Peak & at $1000 \mathrm{~cd} / \mathrm{m}^{2}$ & Peak & at $1000 \mathrm{~cd} / \mathrm{m}^{2}$ \\
\hline $\begin{array}{l}\text { Control } \\
\text { Ligand - }\end{array}$ & 3.8 & 2.5 & 1.8 & 6.1 & 4.3 & 7.2 & 4.1 \\
\hline Exchange & 4.1 & 2.5 & 1.6 & 6.0 & 3.9 & 6.9 & 3.5 \\
\hline
\end{tabular}

\section{Conclusions}

Various synthetic approaches for making CIS QDs were experimented with and results showed that the hot injection of OTT into dissolved $\mathrm{Cu}$ and In precursors was the optimal method due to the high $\eta_{\mathrm{QY}}$ of $14 \%$ for core QDs. OTT most likely improves QD performance due to shorter chain ligands, which increase charge mobility as well it more effectively provides a source of sulfur for the initial reaction. The addition of $\mathrm{ZnS}$ shell in these QDs significantly improved their efficiencies as was expected. By varying the total shell reaction time, two different types of quantum dots were obtained. The longer shell reaction time QDs showed higher $\eta_{\mathrm{QY}}$ reaching $75 \%$ which can be attributed to the thicker shell being more effective at containing charges within the QD layer and also the smoother interfacial area created by the thicker shell. 
QLED devices were successfully fabricated with these QDs and showed significant performance levels with the longer shell reaction QLEDs obtaining $\eta_{\mathrm{A}}$ of $14.7 \mathrm{~cd} / \mathrm{A}$ and $\eta_{\mathrm{EQE}}$ of $5.2 \%$. However, these performance levels still fall short of conventional QLEDs and improvements in efficiency are still needed for viable application of this technology. An attempt at exchanging the oleic acid ligands of the QDs with OTT resulted in only minor changes in QD characteristics and device performance. Further experimentation with ligand exchange procedures and characterization of QDs is needed to determine the full effect of ligand exchange on CIS QDs.

\section{References}

[1]K. Yu, P. Ng, J. Ouyang, M. Zaman, A. Abulrob, T. Baral, D. Fatehi, Z. Jakubek, D. Kingston, X. Wu, X. Liu, C. Hebert, D. Leek and D. Whitfield, "Low-Temperature Approach to Highly Emissive Copper Indium Sulfide Colloidal Nanocrystals and Their Bioimaging Applications", ACS Applied Materials \& Interfaces, vol. 5, no. 8, pp. 2870-2880, 2013.

[2]L. Shi, V. De Paoli, N. Rosenzweig and Z. Rosenzweig, "Synthesis and Application of Quantum Dots FRET-Based Protease Sensors", Journal of the American Chemical Society, vol. 128, no. 32, pp. 10378-10379, 2006.

[3]J. Lott, N. Ledentsov, V. Ustinov, A. Egorov, A. Zhukov, P. Kop'ev, Z. Alferov and D. Bimberg, "Vertical cavity lasers based on vertically coupled quantum dots", Electronics Letters, vol. 33, no. 13, p. 1150, 1997.

[4]H. Shen, W. Cao, N. Shewmon, C. Yang, L. Li and J. Xue, "High-Efficiency, Low Turn-on Voltage Blue-Violet Quantum-Dot-Based Light-Emitting Diodes", Nano Letters, vol. 15, no. 2, pp. 1211 1216, 2015.

[5]D. Vasudevan, R. Gaddam, A. Trinchi and I. Cole, "Core-shell quantum dots: Properties and applications", Journal of Alloys and Compounds, vol. 636, pp. 395-404, 2015.

[6]Y. Shang and Z. Ning, "Colloidal quantum dots surface and device structure engineering for high performance light emitting diodes", National Science Review, p. nww097, 2017.

[7]V. Colvin, M. Schlamp and A. Alivisatos, "Light-emitting diodes made from cadmium selenide nanocrystals and a semiconducting polymer", Nature, vol. 370, no. 6488, pp. 354-357, 1994.

[8]G. Supran, Y. Shirasaki, K. Song, J. Caruge, P. Kazlas, S. Coe-Sullivan, T. Andrew, M. Bawendi and V. Bulović, "QLEDs for displays and solid-state lighting", MRS Bulletin, vol. 38, no. 09, pp. 703$711,2013$.

[9]I. Ugarte, I. Castell, E. Palomares and R. Pacios, "Quantum Dots as a Light Indicator for Emitting Diodes and Biological Coding", Quantum Dots - A Variety of New Applications, 2012.

[10]Y. Shirasaki, G. Supran, M. Bawendi and V. Bulović, "Emergence of colloidal quantum-dot lightemitting technologies", Nature Photonics, vol. 7, no. 1, pp. 13-23, 2013. 
[11]H. Zhang, X. Sun and S. Chen, "Over $100 \mathrm{~cd}$ A-1 Efficient Quantum Dot Light-Emitting Diodes with Inverted Tandem Structure", Advanced Functional Materials, vol. 27, no. 21, p. 1700610, 2017.

[12]"Compliance FAQs: RoHS", NIST, 2018. [Online]. Available: https://www.nist.gov/standardsgov/compliance-faqs-rohs. [Accessed: 11- Apr- 2018].

[13]A. Leach and J. Macdonald, "Optoelectronic Properties of CuInS2 Nanocrystals and Their Origin", The Journal of Physical Chemistry Letters, vol. 7, no. 3, pp. 572-583, 2016.

[14]A. Williams, S. Winfield and J. Miller, "Relative fluorescence quantum yields using a computercontrolled luminescence spectrometer", The Analyst, vol. 108, no. 1290, p. 1067, 1983.

[15]J. Kim and H. Yang, "High-Efficiency Cu-In-S Quantum-Dot-Light-Emitting Device Exceeding 7\%", Chemistry of Materials, vol. 28, no. 17, pp. 6329-6335, 2016.

[16]P. Chuang, C. Lin and R. Liu, "Emission-Tunable CuInS2/ZnS Quantum Dots: Structure, Optical Properties, and Application in White Light-Emitting Diodes with High Color Rendering Index", ACS Applied Materials \& Interfaces, vol. 6, no. 17, pp. 15379-15387, 2014.

[17]L. De Trizio, M. Prato, A. Genovese, A. Casu, M. Povia, R. Simonutti, M. Alcocer, C. D’Andrea, F. Tassone and L. Manna, "Strongly Fluorescent Quaternary Cu-In-Zn-S Nanocrystals Prepared from Cu1-xInS2 Nanocrystals by Partial Cation Exchange", Chemistry of Materials, vol. 24, no. 12, pp. 2400-2406, 2012. 